\title{
The Perineal Membrane: Its Composite Fibers and Nerve Content, and Relationship to the Levator Ani and Deep Transverse Perineal Muscles
}

\author{
Tetsuji Kurokawa1, Nobuyuki Hinata ${ }^{2 *}$, Hiromasa Sasaki ${ }^{3}$, Gen Murakami4,5, \\ Masato Fujisawa ${ }^{2}$, Shin-Ichi Abe ${ }^{5}$, Yoshio Yosida ${ }^{1}$ \\ ${ }^{1}$ Department of Obstetrics and Gynecology, Fukui University School of Medicine, Fukui, Japan \\ ${ }^{2}$ Department of Urology, Kobe University Graduate School of Medicine, Kobe, Japan \\ ${ }^{3}$ Division of Gynecology and Obstetrics, Ishikawa Prefectural Central Hospital, Kanazawa, Japan \\ ${ }^{4}$ Division of Internal Medicine, Iwamizawa Kojin-kai Hospital, Iwamizawa, Japan \\ ${ }^{5}$ Department of Anatomy, Tokyo Dental College, Tokyo, Japan \\ Email: ${ }^{*}$ hinata@med.kobe-u.ac.jp
}

Received 10 April 2014; revised 6 May 2014; accepted 13 May 2014

Copyright (C) 2014 by authors and Scientific Research Publishing Inc.

This work is licensed under the Creative Commons Attribution International License (CC BY).

http://creativecommons.org/licenses/by/4.0/

(c) (i) Open Access

\section{Abstract}

The perineal membrane (PM) is a thick, elastic fiber-rich, smooth muscle-poor membrane extending along the vestibule and lower vaginal wall and embedding the urethrovaginal sphincter and compressor urethrae muscles. To provide a better understanding of the topographical relationship between the PM and the levator ani muscle, we examined histological sections from 15 female cadavers. The composite fibers of the PM were usually continuous with that of a fascia covering the inferior or lateral surface of the levator ani (fascia diaphragmatis pelvis inferior) rather than the endopelvic fascia covering the superior or medial surface of the latter muscle. However, this fascial connection was sometimes interrupted by a venous plexus. The deep transverse perineal muscle was consistently adjacent to the posterolateral aspect of the PM, but whether it extended superficially or deeply to the PM depended on size of the muscle. In contrast to the endopelvic fascia embedding abundant middle-sized nerves (cavernous and sphincter nerves; 0.05 - 0.1 mm in thickness), the PM contained very thin nerves: many in $\mathbf{1 0}$ cadavers but few in 5 cadavers. Most of the nerves seemed to be sensory on the basis of immunohistochemistry. The levator ani muscle was considered likely to provide traction force to the PM, but active elevation appeared to be difficult because of the highly elastic nature of the PM and the interrupting venous plexus. Loss of nerves in the PM might be one of a number of factors that can accelerate pelvic organ prolapse.

${ }^{*}$ Corresponding author.

How to cite this paper: Kurokawa, T., et al. (2014) The Perineal Membrane: Its Composite Fibers and Nerve Content, and Relationship to the Levator Ani and Deep Transverse Perineal Muscles. Open Journal of Obstetrics and Gynecology, 4, 405-415. http://dx.doi.org/10.4236/ojog.2014.47061 


\section{Keywords}

\section{Vagina, Levator Ani Muscle, Perineal Membrane, Smooth Muscles, Elastic Fibers, Nerves}

\section{Introduction}

DeLancey considered the perineal membrane (PM) to be a critical component of level III support [1]. However, in contrast to the perineal body, few studies have attempted to demonstrate the configuration of composite fibers in the membrane-like tissue of the PM. In order for the PM to transfer tensile stress from the vagina to the pelvic floor and vice versa, specific morphological differentiation would be required. On the basis of histologic observations, Stein and DeLancey [2] considered the PM to connect with the endopelvic fascia (fascia pelvis parietalis; a fascia covering the upper or medial surface of the levator ani muscle), but at least on the basis of our interpretation, no such fiber communication was demonstrated. In the same year, Kato et al. clearly demonstrated that elastic fibers between the urethral rhabdosphincter muscle fibers join together to form the female PM [3]. Hinata et al. also demonstrated that the urethrovaginal sphincter, a posterolateral extension of the female urethral rhabdosphincter, is embedded in the PM [4]. Recently, it was reported that the PM ended posteriorly at the perineal smooth muscle mass facing the external anal sphincter [5]. However, none of these studies succeeded in clearly demonstrating the topographical relationship between the PM and the levator ani muscle. In other words, it still seems necessary to evaluate the hypothetical contribution of the endopelvic fascia to PM formation [2]. The primary aim of the present study was to investigate the topographical relationship between the levator ani and the PM.

In classical textbooks of anatomy, the PM has been described as one of the covering fasciae of the deep transverse perineal muscle, i.e., fascia diaphragmatis urogenitale inferior [6]. In contrast, because many researchers have considered that a "urogenital diaphragm" with a striated muscle as a core is absent in females [7] [8], no study appears to have demonstrated the topographical relationship between the deep transverse perineal muscle and the PM in females. However, our standpoint is that the muscle is present in females as well as in males [5]. Therefore, the second aim of this study was to investigate the topographical relationship between the deep transverse perineal muscle and the PM. In addition, in order to clarify the nerves in and around the PM, we conducted immunohistochemistry for neuronal nitric oxide synthase (NOS), vasoactive intestinal peptide (VIP) and tyrosine hydroxylase (TH) in the pelvic autonomic motor nerves [9] [10].

\section{Materials and Methods}

This study was performed in accordance with the provisions of the Declaration of Helsinki [11]. To obtain specimens for histology, we dissected 15 donated female cadavers (all uni- or multiparous women) ranging in age from 79 to 92 years, with a mean age of 86 years. The cause of death had been ischemic heart failure or intracranial bleeding, and we made sure that none of the individuals had undergone surgery by reference to medical documentation as well as macroscopic observation after opening the abdominopelvic cavity. These cadavers had been donated to Tokyo Dental College for research and education on human anatomy, and their use for research had been approved by the university ethics committee. The donated cadavers had been fixed by arterial perfusion with $10 \% \mathrm{v} / \mathrm{v}$ formalin solution and stored in $50 \% \mathrm{v} / \mathrm{v}$ ethanol solution for more than 3 months. From each of the cadavers, we first obtained the entire pelvic tissue mass surrounded by the obturator internus and levator ani muscles. Second, from the tissue mass, we prepared three to five macroslices (15 $\mathrm{mm}$ in thickness) including 1) the urethra, 2) the anterior wall of the vagina and 3) the inferomedial edge of the levator ani. Routine procedures for paraffin-embedded histology were employed, and the sectional planes were frontal (12 cadavers) or horizontal (3 cadavers).

Most sections were stained with hematoxylin and eosin (HE) or Masson trichrome, and some were used for immunohistochemistry as well as elastica Masson staining (a variation of Masson-Goldner staining). Elastica Masson staining is useful for identification of smooth muscles (pink or pale violet), striated muscles (deep red), collagen fibers (green) as well as elastic fibers (black) [10] [12]. The primary antibodies used were 1) mouse monoclonal anti-human S100 protein (1:200 dilution; Dako Z0311; Dako, Glostrup, Denmark) for all nerves, 2) rabbit polyclonal anti-human NOS (1:100; Cell Signaling Technology, Beverly, MA), 3) mouse monoclonal an- 
ti-human VIP (1:00 dilution; Santa Cruz sc25347; Santa Cruz, CA), 4) rabbit polyclonal anti-human TH (1:100; Millipore-Chemicon ab152, Temecula, CA), and 5) mouse monoclonal anti-human alpha smooth muscle actin (1:100; Dako M0851, Glostrup, Denmark) for smooth muscles. Antibodies 2) 3) and 4) covered most of the motor nerves among the pelvic autonomic nerves. The secondary antibody was labeled with horseradish peroxidase (HRP), and antigen-antibody reactions were detected by the HRP-catalyzed reaction with diaminobenzidine. Counterstaining with hematoxylin was performed on the same samples. A negative control without a primary antibody was set up for each of the specimens. Observations and photography were usually performed with a Nikon Eclipse 80, but photos at ultra-low magnification (objective lens less than $\times 2$ ) were taken using a high-grade flat scanner with translucent illumination (Epson scanner GTX970).

\section{Results}

We histologically defined the perineal membrane (PM) as a thick, elastic fiber-rich, smooth muscle-poor membrane extending along the vestibule and lower vaginal wall and embedding the urethrovaginal sphincter and compressor urethrae muscles in accordance with Sasaki et al. [5]. The PM was 0.5 - $5 \mathrm{~mm}$ in thickness: it was thick (or thin) near the external orifice of the urethra (or in the superolateral part). The laminar relationship between the striated sphincter and the PM was easy to identify in horizontal sections (Figure 1) and posteriorly the PM ended at the perineal smooth muscle mass. This smooth muscle mass, corresponding to the lateral extension of the perineal body in Soga et al. (2007), is surrounded by the external anal sphincter, rectum and vagina [5]. In contrast, frontal sections were necessary to examine the topographical relationship of the PM to the levator ani and deep transverse perineal muscles (Figures 2-5). Because composite elastic fibers in the PM were recognized as wavy lines and dots in both horizontal and frontal sections (Figure 1(B) Figure 2(B) Figure 3(C) Figure 4(C) and Figure 5(F)), the PM appeared to be made of a dense mesh of elastic fibers. Smooth muscle fibers were seen in the lateral part of the PM (Figure 2(D) Figure 3(B) and Figure 4(B)), but they disappeared in the medial part near the vagina or external orifice of the urethra. Abundant elastic fibers were sometimes evident between the vestibular bulb and the bulbospongiosus muscle (Figure 5(D)), but their density was lower than in the PM and the layer containing elastic fibers along the external aspect of the vestibular bulb was much thinner than the PM (Figure 5(A)).

The composite fibers of the PM were usually (10 of 12 cadavers for the frontal sections) continuous with that of a fascia covering the inferior or lateral surface of the levator ani muscle, i.e., fascia diaphragmatis pelvis inferior (Figure 2(A) and Figure 4(A)). Thus, the PM, even at the most superior part, was located inferomedial to the inferomedial edge of the levator ani. The connection between the PM and the inferior fascia of the levator ani was sometimes interrupted by a venous plexus. The endopelvic fascia, covering the superior or medial surface of the levator ani muscle, was rich in smooth muscle fibers and merged with the most lateral part of the PM, but the fiber direction and composite fibers were different from those of the PM (Figure 4(B)). The deep transverse perineal muscle was consistently located in the posterolateral side of the PM, but whether it was superficial or deep to the PM varied between specimens: the PM extended between the deep transverse perineal muscle and the vestibular bulb (i.e., in the superficial side of the muscle) when the muscle was well developed (3 of 12 cadavers for frontal sections; Figure 3(A) Figure 3(B)); in 9 of the 12 cadavers, the striated muscle fibers were, to various degrees, embedded in the composite fibers of the lateral part of the PM when the muscle was poorly developed (Figure 2(C) and Figure 4(A)). Thus, the laminar relationship between the muscle and the PM appeared to depend on the size of the muscle.

Abundant medium-sized nerves (0.05 - $0.1 \mathrm{~mm}$ in thickness) were embedded in the endopelvic fascia. These nerves appeared to originate from the distal part of the pelvic plexus in the paracolpium and ran medially and inferiorly toward the urethra and vagina. In contrast, nerves embedded in the PM were very thin ( 0.01 - $0.02 \mathrm{~mm}$ in thickness). As NOS-, VIP- or TH-positive nerve fibers were sparse or absent in the PM, most of the nerves appeared to be sensory. Because the thin nerves in the PM were cut longitudinally or transversely in both horizontal and frontal sections (Figure 1(E) Figure 2(E) and Figure 3(D) insert), a nerve mesh appeared to be present in the PM. Notably, the nerve density in the PM varied considerably between specimens, being high in 10 cadavers (more than 20 cut nerves per $1 \mathrm{~mm}^{2}$ ) or low in 5 cadavers (fewer than 5 cut nerves per $1 \mathrm{~mm}^{2}$ ). Between these two groups of cadavers, we found no difference in the thickness and composite fibers of the PM. In contrast to the PM, the endopelvic fascia (Figure 3(F) and Figure 4(F)) and a site between the levator and rhabdoshincter (Figure 5(B)) contained abundant nerves, i.e., candidates for the cavernous and sphincter nerves. 

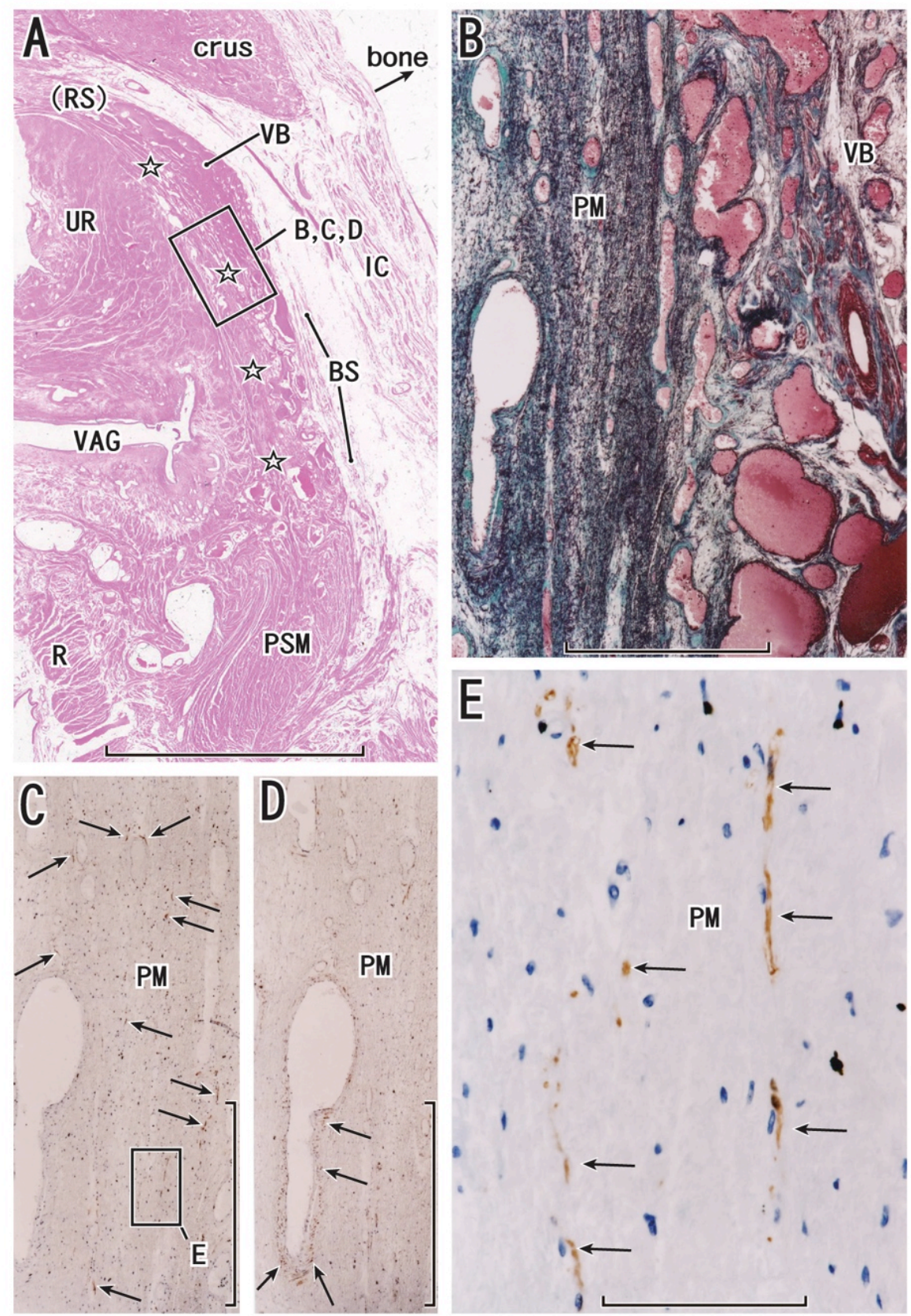

Figure 1. Horizontal sections including the distal urethra and perineal membrane. An 82-year-old woman. Panel A (HE staining) displays the topographical anatomy of the perineal membrane (stars) originating from the inferior end of the urethral rhabdosphincter area (RS with parenthesis), extending between the vestibular bulb (VB) and vaginal wall (VAG) and ending at the perineal smooth muscle mass (PSM). The sciatic bone was removed (bone). Panel B (elastica Masson staining), panel C (immunohistochemistry of S100) and panel D (immunohistochemistry of smooth muscle actin), corresponding to a square in panel A, are higher-magnification views of the perineal membrane (PM). The membrane contains abundant elastic fibers (black lines in panel B) and many thin nerves (arrows in panel C), but smooth muscles are restricted in the vascular wall (arrows in panel D). Panel E (immunohistochemistry of S100), corresponding to the square in panel C, is a higher-magnification view of nerves (arrows) in the perineal membrane. Scale bars: 10 $\mathrm{mm}$ in panel A; $1 \mathrm{~mm}$ in panels $\mathrm{B}, \mathrm{C}$ and $\mathrm{D} ; 0.1 \mathrm{~mm}$ in panel E. BS, bulbospongiosus muscle; crus, crus critoris; IC, ischiocavernosus muscle; R, rectum; UR, urethra. 


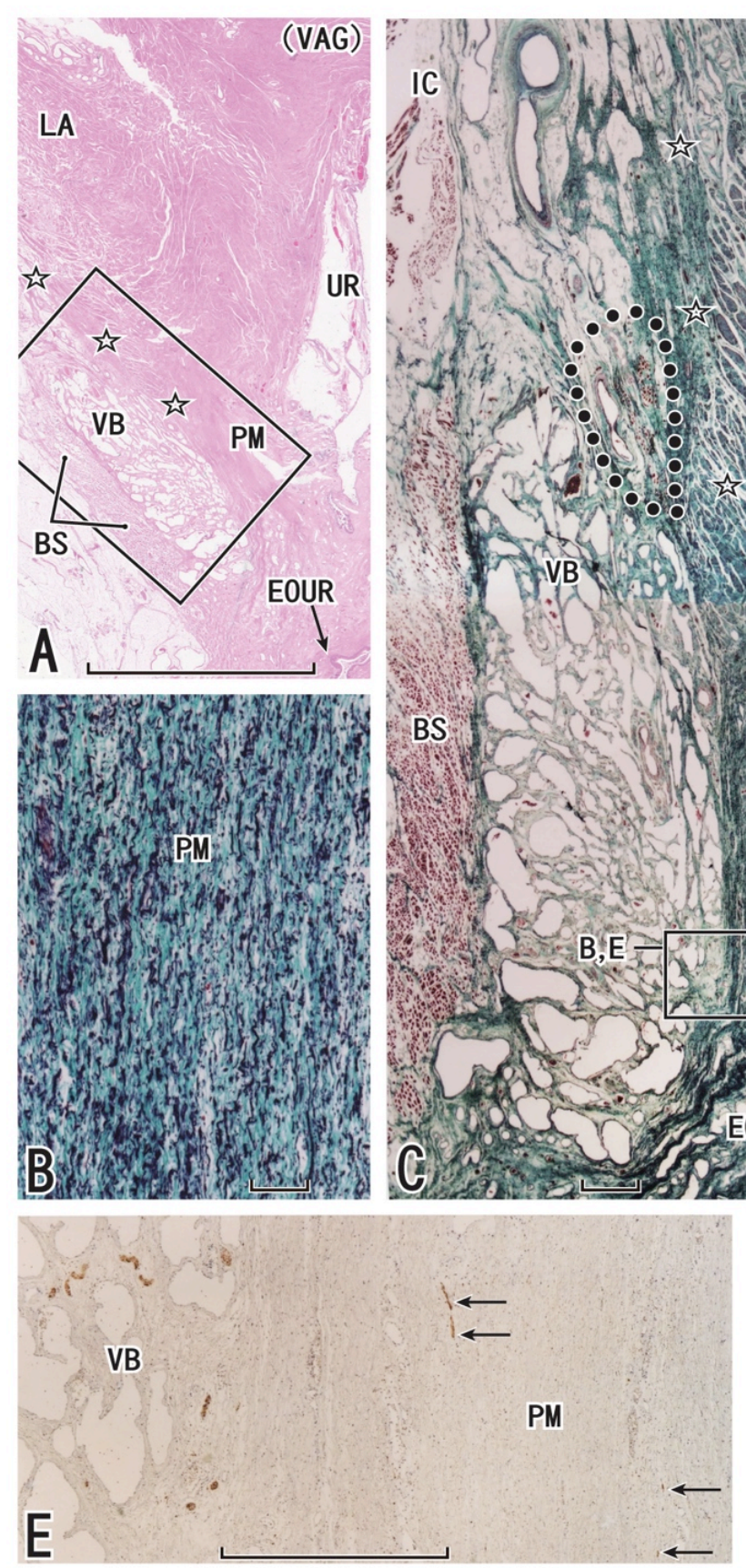

Figure 2. Frontal sections including the distal urethra and perineal membrane. An 86-year-old woman. Panel A (HE staining) displays the topographical anatomy of the perineal membrane (stars, PM) originating from a fascia covering the inferior surface of the levator ani muscle (LA) and extending toward the external orifice of the urethra (EOUR). The anterior wall of the vagina (VAG with parenthesis) is seen in the superior side of the longitudinal section of the urethra (UR). Panel B (elastica Masson staining), corresponding to the square in panel C, displays a higher-magnification view of elastic fibers in the perineal membrane. Panel C (elastica Masson staining) and panel D (immunohistochemistry of smooth muscle actin), corresponding to the square in panel A, exhibit abundant elastic fibers (black fibers in panel C) or a smooth muscle-rich origin of the membrane (stars in panels $\mathrm{C}$ and $\mathrm{D}$ ), respectively. Red muscle fibers encircled by dotted line in panel C represent a candidate for the deep transverse perineal muscle. Panel E (immunohistochemistry of S100), corresponding to the square in panel C, displays few nerves (arrows) contained in the perineal membrane, in contrast to the vestibular bulb (VB). Scale bars: $10 \mathrm{~mm}$ in panel A; $1 \mathrm{~mm}$ in panels $\mathrm{C}$, D and E; $0.1 \mathrm{~mm}$ in panel B. BS, bulbospongiosus muscle; IC, ischiocavernosus muscle; UR, urethra. 


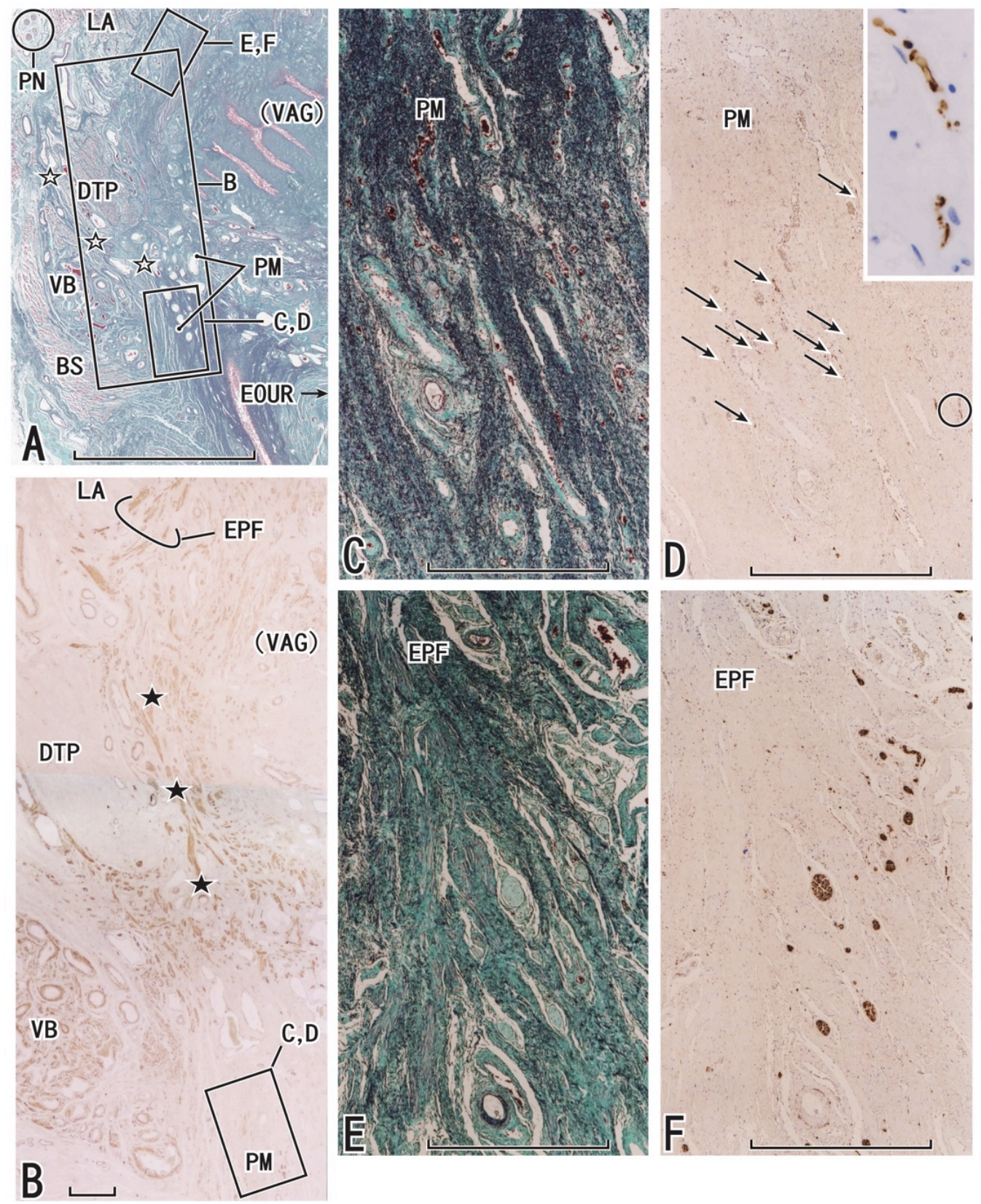

Figure 3. Frontal sections including the external orifice of the urethra as well as the deep transverse perineal muscle. An 81-year-old woman. Panel A (elastica Masson staining) displays the topographical anatomy of the perineal membrane (PM) in which elastic fibers originate from a fascia (open stars) between the deep transverse perineal muscle (DTP) and the vestibular bulb (VB) and extend toward the external orifice of the urethra (EOUR). The anterior wall of the vagina (VAG with parenthesis) can be seen near the inferomedial edge of the levator ani muscle (LA). In panel B (immunohistochemistry for smooth muscle actin), smooth muscles in the endopelvic fascia (EPF) differ in direction from another muscle bundle (black stars) between the deep transverse perineal muscle and the vaginal wall. Panel C (elastica Masson staining) and panel D (immunohistochemistry for S100), corresponding to the square with $\mathrm{CD}$ in panels $\mathrm{A}$ and $\mathrm{B}$, exhibit dense elastic fibers and thin nerves (arrows) in the perineal membrane, respectively. Insert at the upper angle of panel $\mathrm{D}$ is a higher-magnification view of nerves in the circle in panel D. Panel E (elastica Masson staining) and panel F (immunohistochemistry for S100), corresponding to the square with EF in panel A, display elastic fibers (panel E) and nerves (panel F) in the endopelvic fascia (EPF): the density and arrangement differ from those in the perineal membrane. Scale bars: $10 \mathrm{~mm}$ in panel A; $1 \mathrm{~mm}$ in panels B-F. BS, bulbospongiosus muscle; PN, pudendal nerve. 

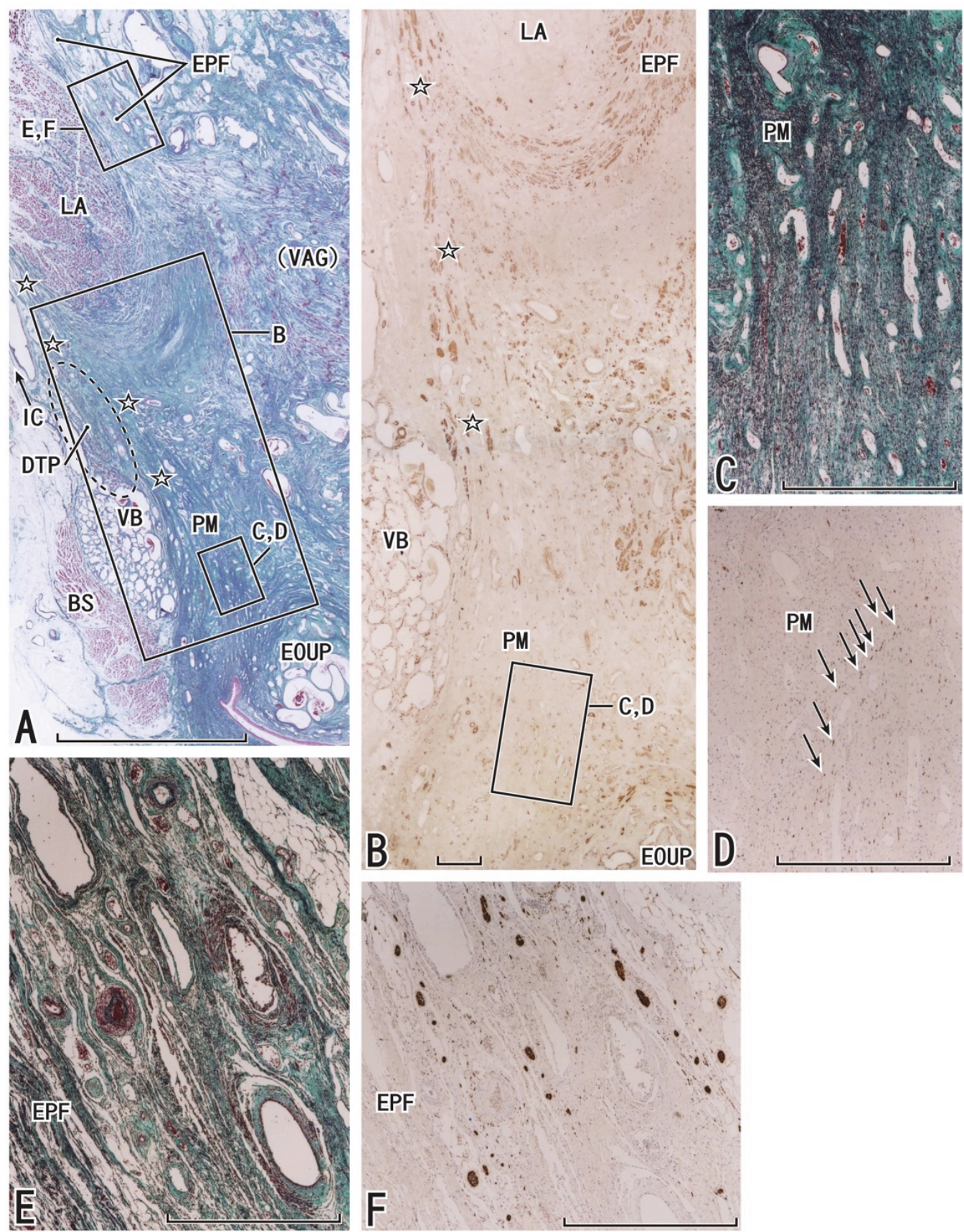

Figure 4. Frontal sections including the external orifice of the urethra as well as the inferomedial edge of the levator ani muscle. An 87-year-old woman. Panel A (elastica Masson staining) displays the topographical anatomy of the perineal membrane (stars, PM) in which elastic fibers originate from a fascia covering the inferior surface of the levator ani (LA) and extend toward the external orifice of the urethra (EOUR). Panel B (immunohistochemistry for smooth muscle actin) displays rich smooth muscles in the endopelvic fascia (EPF) as well as a connection (stars) between the inferior fascia and perineal membrane (PM). Panel C (elastica Masson staining) and panel D (immunohistochemistry of S100), corresponding to the square with CD in panels A and B, exhibit dense elastic fibers and abundant thin nerves (arrows) in the perineal membrane, respectively. Panel E (elastica Masson staining) and panel F (immunohistochemistry of S100), corresponding to the square with EF in panel A, display elastic fibers (panel E) and nerves (panel F) in the endopelvic fascia (EPF): the density and arrangement differ from those in the perineal membrane. Scale bars: $10 \mathrm{~mm}$ in panel A; $1 \mathrm{~mm}$ in panels B-F. BS, bulbospongiosus muscle; DTP, deep transverse perineal muscle; IC, ischiocavernosus muscle. 


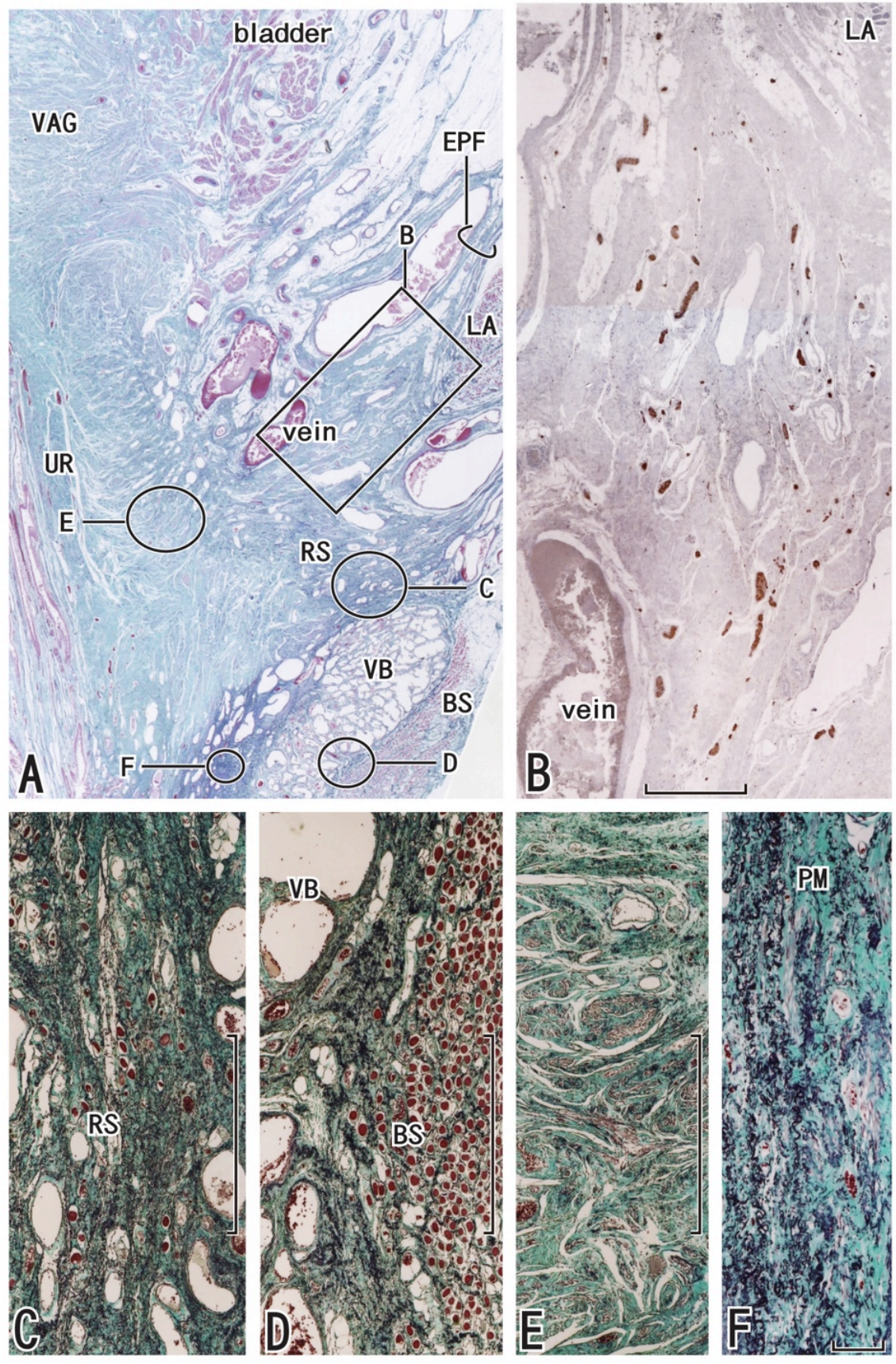

Figure 5. Frontal sections including the urethral rhabdosphincter as well as the inferomedial edge of the levator ani muscle. An 89-year-old woman. Panel A (elastica Masson staining) displays the topographical anatomy of the perineal membrane (stars, $\mathrm{PM}$ ) in which elastic fibers extend laterally toward the inferior or superficial side of the levator ani (LA). Panel B (immunohistochemistry for S100), corresponding to the square in panel A, contains candidates for the cavernous and sphincter nerves. Panels C-E (elastica Masson staining) correspond to the circles in panel A, respectively. The rhabdoshincter area (RS) is overlapped with the perineal membrane: panel C is a higher-magnification view showing the striated muscle fibers (red dots). In panel D, elastic fibers are rich between the vestibular bulb (VB) and the bulbospongiosus muscle (BS). $\mathrm{N}$ panel E, urethral smooth muscles accompany a few elastic fibers. Panel $\mathrm{F}$ is a higher-maginification view of the perineal membrane. Scale bars: $10 \mathrm{~mm}$ in panel A; 1 $\mathrm{mm}$ in panels B-E; $0.1 \mathrm{~mm}$ in panel F. 


\section{Discussion}

The present study clearly demonstrated the topographical relationship of the elastic fiber-rich smooth muscle-poor PM with the inferomedial edge of the levator ani muscle. Although various amounts of smooth muscle have been reported in the PM [13], these might have represented the lateral or superior part. Previous data on the thickness of the PM (mean, $5.5 \mathrm{~mm}$ ) [13] had suggested that it was much thicker than in the present series of specimens (5.5 mm at maximum), but this difference may have simply been attributable to the definition employed, i.e. macroscopic or histologic. The composite fibers of the PM were usually continuous with that of a fascia covering the inferior or lateral surface of the levator ani (fascia diaphragmatis pelvis inferior). This morphology was different from the endopelvic fascia covering the superior or medial surface of the levator ani and joining the external longitudinal smooth muscle layer of the rectum to form the longitudinal anal muscle [5] [14]. As an elastic fiber plate, the PM might be the second largest or longest of its type in the body: the longest is the aortic media, although its elastic fibers cooperate with smooth muscles [15].

Elastic fiber-rich connective tissues have been well described in the larynx [16] [17], the middle ear [18] and the urethra [19]. According to Hinata et al. [19], the male urethra contains a much greater amount of elastic fibers than the female urethra. At these three sites, in combination with hyaluronic acid as a physiological lubricant, elastic fibers seem to avoid damage at their points of connection under conditions of vibration or drastic changes in shape. Elastic fibers in the PM are also likely to play a critical role in recovery of the topographical relationship of the vagina with the external orifice of the urethra after vaginal delivery and sexual activity. In addition, due to the close topography, the PM would appear to accelerate recovery of the size and shape of the cavernous tissues, especially the vestibular bulb. The levator ani muscle is likely to provide traction force for the PM via the inferior fascia of the muscle, but any active effect such as elevation would appear to be difficult because of the highly elastic nature of the PM.

As typically seen in extremities, the covering fascia of a striated muscle contains few or no elastic fibers, because its elastic nature is due largely to the mesh structure of its constituent collagen fibers [20] and partly to elasticity of the muscle cells themselves [21]. Striated muscle in a fascia functions to provide mechanical stress, which specifies the configuration of the fascial structure [21] [22]. As the PM incorporates the striated sphincters of the urethra and vagina, and sometimes the deep transverse perineal muscle, it appears not to be a "fascia of striated muscle". In males, the deep transverse perineal muscle is not sheet-like but a 3-dimensional pillar continuous with the urethral striated sphincter [23]. Thus, these muscles appeared to join together and correspond to a mother muscle of the distinct fascia, i.e., the PM. The PM connected the deep transverse perineal muscle to the vestibule, whereas the endopelvic fascia connects the levator ani muscle to the rectum [5] [14]. In this context, the PM as well as the endopelvic fascia might be considered a specific fascia for pelvic floor support. Table 1 shows the similarities and differences between these two fasciae. The smooth muscle-rich endopelvic fascia is likely to contract in cooperation with the levator ani muscle under nerve control, whereas the elastic fiber-rich PM is unlikely to contract.

In the present study, the endopelvic fascia contained abundant medium-sized nerves, which were likely to be the cavernous and sphincter nerves on the basis of male anatomy [24] [25]. Some of these nerves extend to the interface between the vagina and the levator ani [10], despite the tensile stress that would be expected. The paravaginal nerves are characterized by a rich content of VIP-positive nerves [9] [26]. In contrast, the nerves in the $\mathrm{PM}$, much thinner than those in the endopelvic fascia and paracolpium, appear to be sensory-dominant. This nerve morphology is similar to that in the perineal smooth muscles including the perineal body [5]. As is the case for the fine nerve mesh in tendons of striated muscles of the extremity [27], changes in the mechanical condition of the PM may be input to the spinal cord. Heterogeneity of connective tissue according to mechanical stress is likely to be maintained by the sensory nerves [28]. Notably, in 5 of the cadavers we examined, the PM carried few nerves. Sensory innervation would appear to favor connective tissue repair [29] [30]. Conversely, the suggested loss of nerves in the PM might be one of multiple factors that accelerate pelvic organ prolapse.

Table 1. Comparison between the perineal membrane and endopelvic fascia.

\begin{tabular}{ccccc} 
& Elastic fibers & Smooth muscles & Nerves contained & Mother muscle \\
\hline Perineal Membrane & ++ & \pm & Many or few, very thin & DTP \& sphincter \\
Endopelvic Fascia & + & ++ & Many, medium-sized & Levator ani muscle \\
\hline
\end{tabular}


The PM has often been described as a macroscopic entity in accounts of the course of the perineal nerve [31]-[33]. The inferior fascia of the urogenital diaphragm is known as the PM, while the superior fascia is known as Colles' fascia [6]. However, discrimination between these fasciae seems to be difficult, even in males [12] [23]. Because the deep transverse perineal muscle is often poorly developed and embedded in the PM in females, discrimination of these fasciae is often impossible. We suspect that, in terms of gross anatomy, the two perineal fasciae, i.e., Colles' fascia and the PM, might be a result of 1) overestimation after forced separation of the bulky vestibular bulb from the levator ani during dissection and/or 2) a dissection artifact, as Range and Woodburne [34] and Kato et al. [35] have hypothesized for the cardinal ligament of the uterus.

\section{Acknowledgements}

We are grateful to the individuals who donated their bodies after death to Tokyo Dental College for research and education on human anatomy without any socio-economical benefit. We also thank their families for agreeing to the donation, as well as their patience in waiting for return of their loved ones' remains after study.

\section{References}

[1] DeLancey, J.O. (1999) Structural Anatomy of the Posterior Pelvic Compartment as It Relates to Rectocele. American Journal of Obstetrics \& Gynecology, 180, 815-823. http://dx.doi.org/10.1016/S0002-9378(99)70652-6

[2] Stein, T.A. and DeLancey, J.O. (2008) Structure of the Perineal Membrane in Females: Gross and Microscopic Anatomy. Obstetrics \& Gynecology, 111, 686-893. http://dx.doi.org/10.1097/AOG.0b013e318163a9a5

[3] Kato, M., Matsubara, A., Murakami, G., et al. (2008) Female Perineal Membrane: A Study Using Pelvic Floor Semiserial Sections from Elderly Nulliparous and Multiparous Women. International Urogynecology Journal, 19, 1663-1670. http://dx.doi.org/10.1007/s00192-008-0701-0

[4] Hinata, N., Murakami, G., Abe, S., et al. (2012) Detailed Histological Investigation of the Female Urethra: Application to Radical Cystectomy. The Journal of Urology, 187, 451-456. http://dx.doi.org/10.1016/j.juro.2011.10.037

[5] Sasaki, H., Hinata, N., Kurokawa, T. and Murakami, G. (2014) Supportive Tissues of the Vagina with Special Reference to a Fibrous Skeleton in the Perineum: A Review. Open Journal of Obstetrics and Gynecology, 144-157.

[6] Standring, S. (2008) Gray’s Anatomy: The Anatomical Basis of Clinical Practice. Churchill Livingstone/Elsevier, Edinburgh.

[7] Oelrich, T.M. (1983) The Striated Urogenital Sphincter Muscle in the Female. The Anatomical Record, 205, $223-232$. http://dx.doi.org/10.1002/ar.1092050213

[8] Fritsch, H., Lienemann, A., Brenner, E. and Ludwikowski, B. (2004) Clinical Anatomy of the Pelvic Floor. Advances in Anatomy, Embryology and Cell Biology, 175, 1-64. http://dx.doi.org/10.1007/978-3-642-18548-9

[9] Hinata, N., Hieda, K., Sasaki, H., et al. (2014) Topohistology of Sympathetic and Parasympathetic Nerve Fibers in Branches of the Pelvic Plexus: An Immunohistochemical Study Using Donated Elderly Cadavers. Anatomy \& Cell Biology, 47, 55-65. http://dx.doi.org/10.5115/acb.2014.47.1.55

[10] Hinata, N., Hieda, K., Sasaki, H., et al. (2014) Nerves and Fasciae in and around the Paracolpium or Paravaginal Tissue: An Immunohistochemical Study Using Elderly Donated Cadavers. Anatomy \& Cell Biology, 47, 44-54. http://dx.doi.org/10.5115/acb.2014.47.1.44

[11] World Medical Association (2013) World Medical Association Declaration of Helsinki: Ethical Principles for Medical Research Involving Human Subjects. Journal of the American Medical Association, 310, 2191-2194. http://dx.doi.org/10.1001/jama.2013.281053

[12] Hinata, N. and Murakami, G. (2014) The Urethral Rhabdosphincter, Levator Ani Muscle, and Perineal Membrane: A Review. BioMed Research International, 2014, Article ID: 906921.

[13] Betschart, C., Scheiner, D., Maake, C., et al. (2008) Histomorphological Analysis of the Urogenital Diaphragm in Elderly Women: A Cadaver Study. International Urogynecology Journal, 19, 1477-1481. http://dx.doi.org/10.1007/s00192-008-0669-9

[14] Arakawa, T., Murakami, G., Nakajima, F., et al. (2004) Morphologies of the Interfaces between the Levator Ani Muscle and Pelvic Viscera, with Special Reference to Muscle Insertion into the Anorectum in Elderly Japanese. Anatomical Science International, 79, 72-81. http://dx.doi.org/10.1111/j.1447-073x.2004.00069.x

[15] Dingemans, K.P., Teeling, P., Lagendijk, J.H. and Becker, A.E. (2000) Extracellular Matrix of the Human Aortic Media: An Ultrastructural Histochemical and Immunohistochemical Study of the Adult Aortic Media. The Anatomical Record, 258, 1-14. http://dx.doi.org/10.1002/(SICI)1097-0185(20000101)258:1<1::AID-AR1>3.0.CO;2-7

[16] Hahn, M.S., Kobler, J.B., Starcher, B.C., Zeitels, S.M. and Langer, R. (2006) Quantitative and Comparative Studies of 
the Vocal Fold Extracellular Matrix. I: Elastic Fibers and Hyaluronic Acid. Annals of Otology, Rhinology, and Laryngology, 115, 156-164.

[17] Korn, G.P., Martins, J.R., Park, S.W., Mendes, A., Kobayashi, E.Y., Nader, H.B., et al. (2011) Concentration of Hyaluronic Acid in Human Vocal Folds in Young and Old Subjects. Otolaryngology_Head and Neck Surgery, 145, 981986. http://dx.doi.org/10.1177/0194599811419457

[18] Kawase, T., Shibata, S., Katori, Y., Ohtsuka, A., Murakami, G. and Fujimiya, M. (2012) Elastic Fiber-Mediated Enthesis in the Human Middle Ear. Journal of Anatomy, 221, 331-340. http://dx.doi.org/10.1111/j.1469-7580.2012.01542.x

[19] Hinata, N., Murakami, G., Abe, S., Shibata, S., Morizane, S., Honda, M., et al. (2013) Coexistence of Elastic Fibers with Hyaluronic Acid in the Human Urethral Sphincter Complex: A Histological Study. The Journal of Urology, 190, 1313-1319. http://dx.doi.org/10.1016/j.juro.2013.04.023

[20] Stecco, C., Porzionato, A., Macchi, V., Tiengo, C., Parenti, A., Aldegheri, R., et al. (2006) Histological Characteristics of the Deep Fascia of the Upper Limb. Italian Journal of Anatomy and Embryology, 111, 105-110.

[21] Ochala, J., Frontera, W.R., Dorer, D.J., Van Hoecke, J. and Krivickas, L.S. (2007) Single Skeletal Muscle Fiber Elastic and Contractile Characteristics in Young and Older Men. The Journals of Gerontology Series A: Biological Sciences and Medical Sciences, 62, 375-381. http://dx.doi.org/10.1016/j.juro.2013.04.023

[22] Hayes, M.A. (1950) Abdominopelvic Fasciae. American Journal of Anatomy, 87, 119-161. http://dx.doi.org/10.1002/aja.1000870105

[23] Nakajima, F., Takenaka, A., Uchiyama, E., Hata, F., Suzuki, D. and Murakami, G. (2007) Macroscopic and Histotopographic Study of the Deep Transverse Perineal Muscle (Musculus Transversus Perinei Profundus) in Elderly Japanese. Annals of Anatomy, 189, 65-74. http://dx.doi.org/10.1002/aja.1000870105

[24] Takenaka, A., Kawada, M., Murakami, G., Hisasue, S., Tsukamoto, T. and Fujisawa, M. (2005) Interindividual Variation in Distribution of Extramural Ganglion Cells in the Male Pelvis: A Semi-Quantitative and Immunohistochemical Study Concerning Nerve-Sparing Pelvic Surgery. European Urology, 48, 46-52.

[25] Takenaka, A., Hara, R., Soga, H., Murakami, G. and Fujisawa, M. (2005) A Novel Technique for Approaching the Endopelvic Fascia in Retropubic Radical Prostatectomy, Based on an Anatomical Study of Fixed and Fresh Cadavers. BJU International, 95, 766-771. http://dx.doi.org/10.1111/j.1464-410X.2005.05397.X

[26] Hoyle, C.H., Stones, R.W., Robson, T., Whitley, K. and Burnstock, G. (1996) Innervation of Vasculature and Microvasculature of the Human Vagina by NOS and Neuropeptide-Containing Nerves. Journal of Anatomy, 188, 633-644.

[27] Andres, K.H., von Düring, M. and Schmidt, R.F. (1985) Sensory Innervation of the Achilles Tendon by Group III and IV Afferent Fibers. Anatomy and Embryology (Berlin), 172, 145-156. http://dx.doi.org/10.1007/BF00319597

[28] Arruda, E.M., Calve, S, Dennis, R.G., Mundy, K. and Baar, K. (2006) Regional Variation of Tibialis Anterior Tendon Mechanics Is Lost Following Denervation. Journal of Applied Physiology, 101, 1113-1117. http://dx.doi.org/10.1152/japplphysiol.00612.2005

[29] Ackermann, P.W., Ahmed, M. and Kreicbergs, A. (2002) Early Nerve Regeneration after Achilles Tendon Rupture-A Prerequisite for Healing? A Study in the Rat. Journal of Orthopaedic Research, 20, 849-856. http://dx.doi.org/10.1016/S0736-0266(01)00159-0

[30] Carlsson, O., Schizas, N., Li, J. and Ackermann, P.W. (2011) Substance P Injections Enhance Tissue Proliferation and Regulate Sensory Nerve Ingrowth in Rat Tendon Repair. Scandinavian Journal of Medicine and Science in Sports, 21, 562-569. http://dx.doi.org/10.1111/j.1600-0838.2009.01080.x

[31] Bonnet, P., Waltregny, D., Reul, O. and de Leval, J. (2005) Transobturator Vaginal Tape Inside out for the Surgical Treatment of Female Stress Urinary Incontinence: Anatomical Considerations. The Journal of Urology, 173, 12231228. http://dx.doi.org/10.1097/01.ju.0000148364.13525.7b

[32] Vaze, A., Goldman, H., Jones, J.S., Rackley, R., Vasavada, S. and Gustafson, K.J. (2008) Determining the Course of the Dorsal Nerve of the Clitoris. Urology, 72, 1040-1043. http://dx.doi.org/10.1016/j.urology.2008.07.029

[33] Montoya, T.I., Calver, L., Carrick, K.S., Prats, J. and Corton, M.M. (2011) Anatomic Relationships of the Pudendal Nerve Branches. American Journal of Obstetrics and Gynecology, 205, 504.

[34] Range, R.L. and Woodburne, R.T. (1964) The Gross and Microscopic Anatomy of the Transverse Cervical Ligament. American Journal of Obstetrics and Gynecology, 90, 460-467.

[35] Kato, T., Murakami, G. and Yabuki, Y. (2002) Does the Cardinal Ligament of the Uterus Contain a Nerve That Should Be Preserved in Radical Hysterectomy? Anatomical Science International, 77, 161-168. http://dx.doi.org/10.1046/j.0022-7722.2002.00023.x 